University of Nebraska - Lincoln

DigitalCommons@University of Nebraska - Lincoln

Faculty Publications, Department of Physics and

Astronomy

Research Papers in Physics and Astronomy

2009

Atomic Spectral-Product Representations of Molecular Electronic Structure: Metric Matrices and Atomic-Product Composition of Molecular Eigenfunctions

M.Ben-Nun

J.D. Mills

R. J. Hinde

C. L. Winstead

J.A. Boatz

See next page for additional authors

Follow this and additional works at: https://digitalcommons.unl.edu/physicsfacpub

This Article is brought to you for free and open access by the Research Papers in Physics and Astronomy at DigitalCommons@University of Nebraska Lincoln. It has been accepted for inclusion in Faculty Publications, Department of Physics and Astronomy by an authorized administrator of DigitalCommons@University of Nebraska - Lincoln. 
Authors

M. Ben-Nun, J. D. Mills, R. J. Hinde, C. L. Winstead, J. A. Boatz, G. A. Gallup, and P. W. Langhoff 


\title{
Atomic Spectral-Product Representations of Molecular Electronic Structure: Metric Matrices and Atomic-Product Composition of Molecular Eigenfunctions ${ }^{\dagger}$
}

\author{
M. Ben-Nun, ${ }^{\ddagger}$ J. D. Mills, ${ }^{\S}$ R. J. Hinde,, C. L. Winstead, ${ }^{\perp}$ J. A. Boatz, ${ }^{\S}$ G. A. Gallup, ${ }^{\#}$ and \\ P. W. Langhoff*, \\ San Diego Supercomputer Center and Department of Chemistry \& Biochemistry, University of California, 9500 \\ Gilman Drive, La Jolla, California 92093-0505, Air Force Research Laboratory, 10 East Saturn Blvd., \\ Edwards AFB, California 93524-7680, Department of Chemistry, University of Tennessee, Knoxville, Tennessee \\ 37996-1600, A. A. Noyes Laboratory of Chemical Physics, California Institute of Technology, Pasadena, \\ California 91125, and Department of Physics and Astronomy, University of Nebraska, Lincoln, Nebraska \\ 68588-0111
}

Received: February 16, 2009; Revised Manuscript Received: April 30, 2009

\begin{abstract}
Recent progress is reported in development of ab initio computational methods for the electronic structures of molecules employing the many-electron eigenstates of constituent atoms in spectral-product forms. The approach provides a universal atomic-product description of the electronic structure of matter as an alternative to more commonly employed valence-bond- or molecular-orbital-based representations. The Hamiltonian matrix in this representation is seen to comprise a sum over atomic energies and a pairwise sum over Coulombic interaction terms that depend only on the separations of the individual atomic pairs. Overall electron antisymmetry can be enforced by unitary transformation when appropriate, rather than as a possibly encumbering or unnecessary global constraint. The matrix representative of the antisymmetrizer in the spectralproduct basis, which is equivalent to the metric matrix of the corresponding explicitly antisymmetric basis, provides the required transformation to antisymmetric or linearly independent states after Hamiltonian evaluation. Particular attention is focused in the present report on properties of the metric matrix and on the atomic-product compositions of molecular eigenstates as described in the spectral-product representations. Illustrative calculations are reported for simple but prototypically important diatomic $\left(\mathrm{H}_{2}, \mathrm{CH}\right)$ and triatomic $\left(\mathrm{H}_{3}, \mathrm{CH}_{2}\right)$ molecules employing algorithms and computer codes devised recently for this purpose. This particular implementation of the approach combines Slater-orbital-based one- and two-electron integral evaluations, valence-bond constructions of standard tableau functions and matrices, and transformations to atomic eigenstateproduct representations. The calculated metric matrices and corresponding potential energy surfaces obtained in this way elucidate a number of aspects of the spectral-product development, including the nature of closure in the representation, the general redundancy or linear dependence of its explicitly antisymmetrized form, the convergence of the apparently disparate atomic-product and explicitly antisymmetrized atomic-product forms to a common invariant subspace, and the nature of a chemical bonding descriptor provided by the atomicproduct compositions of molecular eigenstates. Concluding remarks indicate additional studies in progress and the prognosis for performing atomic spectral-product calculations more generally and efficiently.
\end{abstract}

\section{Introduction}

The electronic eigenstates of atoms provide a well-known formal basis for outer-product representations of molecular electronic wave functions and calculations of interaction energies in long-range separation limits. ${ }^{1}$ More generally, ab initio investigations of the electronic structures and associated potential energy surfaces of molecules as currently performed tend to employ explicitly antisymmetric representations based on early molecular-orbital ${ }^{2,3}$ or valence-bond ${ }^{4,5}$ descriptions of the relevant electronic degrees of freedom. ${ }^{6,7}$ In these approaches, the presence of the component atomic constituents is not made explicit but is evident largely in the choices of atomic orbitals

\footnotetext{
"Part of the "Robert Benny Gerber Festschrift".

* Corresponding author. E-mail: langhoff@drifter.sdsc.edu. Fax: 858534-4974. Phone: 858-822-3611.

* University of California.

$\S$ Air Force Research Laboratory.

"University of Tennessee.

${ }^{\perp}$ California Institute of Technology.

\# University of Nebraska.
}

employed in the many-electron molecular representational basis sets. It has proved difficult to base a general ab initio computational approach to the electronic structures of molecules directly on an atomic eigenstate-product representation, ${ }^{8,9}$ in spite of the fact that atoms clearly comprise molecules and other forms of matter, upon which recognition the conceptual foundations of chemistry largely rest.

A recent series of reports motivated by earlier work emphasizes both the difficulties and potential advantages of adopting an atomic eigenstate-product representation in ab initio calculations of molecular electronic structures. ${ }^{10-14}$ This approach, which has been described in considerable detail in the previous reports, is predicated largely on the demonstrable closure of the outer spectral product of complete constituent atomic eigenstates for representations of molecular electronic states in the absence of explicit (term-by-term) enforcement of aggregate electron antisymmetry. Although both conceptual and computational barriers must be overcome in such a universal atomicfragment-based approach to molecular electronic structure, 
certain potential advantages follow from its adoption. These include the possibility of performing atomic and atomicinteraction calculations employing highly accurate configurationinteraction methods and retaining such information for repeated molecular applications, employing entirely analytical methods in calculating the angular dependences of atomic interactions, and the opportunity to enforce molecular antisymmetry when required, rather than as a possibly encumbering overall global constraint, to mention some important potential advantages. ${ }^{9-14}$

In the present contribution, additional theoretical aspects of the atomic spectral-product approach to molecular electronic structure are reported, the essential features of a recently devised computer code suite for performing such calculations are indicated, and selected computational applications to prototypically important diatomic $\left(\mathrm{H}_{2}, \mathrm{CH}\right)$ and triatomic $\left(\mathrm{H}_{3}, \mathrm{CH}_{2}\right)$ molecules are presented. Calculations of the metric matrices in these cases, and of corresponding potential energy curves and surfaces, in spectral-product representations illustrate the capabilities of the Slater-orbital- and valence-bond-based algorithms and codes devised. The metric matrices, which provide a connecting bridge between spectral-product representations in the absence or presence of term-by-term antisymmetry, clarify a number of aspects of the development, including the nature of closure in the two representations and the general redundancy of the explicitly antisymmetrized form. The calculations of the eigenspectrum of the antisymmetrizer in the spectral-product representation reported for $\mathrm{H}_{2}$ and $\mathrm{H}_{3}$ demonstrate quantitatively its nature as a projection-operator and illustrate the additional redundancies which arise between commonly employed chargetransfer and one-electron covalent excitations in these cases. The corresponding potential energy surfaces for ground and excited states of $\mathrm{H}_{2}$ and $\mathrm{H}_{3}$ illustrate the capabilities of the Slaterorbital-based integral evaluations employed in the codes devised. The atomic-product compositions of the spectral-product representations of molecular eigenfunctions are seen to provide novel quantitative descriptors of chemical bonding in $\mathrm{CH}$ and $\mathrm{CH}_{2}$ molecules, which have played important historical roles in the development of accurate ab initio quantum methods for electronic structure and spectra. ${ }^{15,16}$

The theory is reviewed briefly in section II, where the atomic spectral representations of aggregate electronic degrees of freedom are defined, ${ }^{9,10}$ the forms of metric and Hamiltonian matrices with and without explicit antisymmetrization are reported, ${ }^{11,12}$ and the unitary transformation formalism for isolation of the physical and linearly independent Hamiltonian subspaces are given and their equivalence established. ${ }^{13,14}$ Calculations employing the recently devised computer codes, reported in section III, include metric matrices and energy eigenvalues for the $\mathrm{H}_{2}, \mathrm{CH}, \mathrm{H}_{3}$, and $\mathrm{CH}_{2}$ molecules. Discussion and concluding remarks presented in section IV describe additional studies in progress and strategies employed in performing atomic spectral-product calculations more generally and efficiently.

\section{Theoretical Background and Developments}

Selected background information is provided in this section in summary of previously devised aspects of the spectral-product approach to molecular electronic structure, ${ }^{9-14}$ and new theoretical developments and clarifications are reported. The atomic spectral-product representations of molecular electronic states of interest here are described in section A, construction of Hamiltonian matrices in these representations are described in section $\mathrm{B}$, and the role of the aggregate metric matrix in isolation of physically significant Schrödinger eigenstates is described in section $\mathrm{C}$.
A. Atomic Spectral-Product Representations. The adiabatic (Born-Oppenheimer) electronic states obtained from solution of the nonrelativistic Schrödinger equation

$$
\hat{H}(\mathbf{r}: \mathbf{R}) \Psi(\mathbf{r}: \mathbf{R})=\Psi(\mathbf{r}: \mathbf{R}) \cdot \mathbf{E}(\mathbf{R})
$$

provide useful first approximations to the chemical and physical attributes of molecules and other atomic aggregates. ${ }^{17}$ Here, the row vector $\Psi(\mathbf{r}: \mathbf{R})$ contains the desired eigenstates, the diagonal matrix $\mathbf{E}(\mathbf{R})$ contains the corresponding energy eigenvalues, $\mathbf{r}$ refers to the coordinates of the $n_{t}$ electrons of the system, and $\mathbf{R}$ designates the fixed spatial positions $\left(\mathbf{R}_{1}, \mathbf{R}_{2}, \ldots, \mathbf{R}_{N}\right)$ of the $N$ atomic nuclei.

The nonrelativistic Hamiltonian operator in eq 1,

$$
\begin{aligned}
\hat{H}(\mathbf{r}: \mathbf{R})=\sum_{k=1}^{n_{t}}-\frac{\hbar^{2}}{2 m} \nabla_{k}^{2}-\sum_{\alpha=1}^{N} \sum_{k=1}^{n_{t}} \frac{Z_{\alpha} e^{2}}{r_{k \alpha}}+ \\
\sum_{k=1}^{n_{t}-1} \sum_{k^{\prime}=k+1}^{n_{t}} \frac{e^{2}}{r_{k k^{\prime}}}+\sum_{\alpha=1}^{N-1} \sum_{\beta=\alpha+1}^{N} \frac{Z_{\alpha} Z_{\beta} e^{2}}{R_{\alpha \beta}}
\end{aligned}
$$

contains the familiar kinetic, electron-nuclear attraction, and electron and nuclear repulsion operators and is totally symmetric in all electron coordinates. ${ }^{18}$ Consequently, the solutions of eq 1 can be classified according to the irreducible representations of the symmetric group $\mathbf{S}_{n_{t}}$, with the totally antisymmetric solutions providing the physically significant Schrödinger eigenstates. $^{19}$

The atomic spectral-product representation is written in the outer-product $(\otimes)$ form ${ }^{9,10}$

$$
\boldsymbol{\Phi}(\mathbf{r}: \mathbf{R})=\left\{\boldsymbol{\Phi}^{(1)}(\mathbf{1}) \otimes \boldsymbol{\Phi}^{(2)}(\mathbf{2}) \otimes \cdots \boldsymbol{\Phi}^{(N)}(\boldsymbol{n})\right\}_{\mathrm{O}}
$$

where the row vectors $\boldsymbol{\Phi}^{(\alpha)}(\mathbf{i})$ contain the formally complete orthonormal antisymmetric eigenstates of the constituent atoms ( $\alpha=1$ to $N$ ) specified by the standard atomic quantum numbers $\left(E, L, M_{L}, S, M_{S}, P\right)_{\alpha},{ }^{20}$ with $\mathbf{i}$ referring to the coordinates of the $n_{\alpha}$ electrons arbitrarily assigned to the atom $\alpha$ measured relative to the atomic centers $\mathbf{R}_{\alpha}$. These atomic states can be calculated employing any of a number of highly efficient modern computational methods, including highly accurate configurationinteraction approaches. ${ }^{17}$ The subscript " $\mathrm{O}$ " in eq 3 indicates the adoption of a particular ordering convention for the vector sequence of spectral-product functions employed. More detailed descriptions of these and of the other notational conventions of eqs $1-3$ and in the subsequent text have been reported previously elsewhere..$^{9-14}$

Although the representation of eq 3 is not term-by-term antisymmetric in all electron coordinates, it is, in fact, complete in the limit of spectral closure for representations of totally antisymmetric aggregate eigenstates. ${ }^{1,13,21}$ Accordingly, eq 3 is in this connection formally equivalent to the more familiar explicitly (term-by-term) antisymmetric form ${ }^{8}$

$$
\begin{aligned}
\boldsymbol{\Phi}_{\mathrm{A}}(\mathbf{r}: \mathbf{R}) \equiv \hat{P}_{\mathrm{A}} \boldsymbol{\Phi}(\mathbf{r}: \mathbf{R})= \\
\hat{P}_{\mathrm{A}}\left\{\boldsymbol{\Phi}^{(1)}(\mathbf{1}) \otimes \boldsymbol{\Phi}^{(2)}(\mathbf{2}) \otimes \cdots \Phi^{(N)}(\boldsymbol{n})\right\}_{\mathrm{O}}
\end{aligned}
$$




$$
\hat{P}_{\mathrm{A}} \equiv\left(n_{t} !\right)^{-1 / 2}\left(n_{1} ! n_{2} ! \cdots n_{N} !\right)^{-1 / 2} \sum_{p=1}^{n_{t} !}(-1)^{\delta_{p}} \hat{P}_{p}
$$

is the aggregate antisymmetrizer, ${ }^{19}$ normalized to ensure that $\left\langle P_{\mathrm{A}} \mathbf{\Phi}(\mathbf{r}: \mathbf{R}) \mid P_{\mathrm{A}} \boldsymbol{\Phi}(\mathbf{r}: \mathbf{R})\right\rangle$ becomes the unit matrix $(\rightarrow \mathbf{I})$ in the limit $(\mathbf{R} \rightarrow \infty)$, in accordance with the orthonormality of the squareintegrable antisymmetric atomic eigenstates in the row vectors $\boldsymbol{\Phi}^{(\alpha)}(\mathbf{i})$. Alternatively, it is possible to enforce aggregate electron antisymmetry employing a so-called coset decomposition of the antisymmetrizer to isolate those electron permutations not completely internal to the already antisymmetric atomic eigenstates. ${ }^{8,22}$

An important difference between the representations of eqs 3 and 4 is found in their respective closure relations. In the case of the orthonormal representation of eq 3 , the closure expression is $\boldsymbol{\Phi}(\mathbf{r}: \mathbf{R}) \cdot \boldsymbol{\Phi}\left(\mathbf{r}^{\prime}: \mathbf{R}\right)^{\dagger}=\delta\left(\mathbf{r}-\mathbf{r}^{\prime}: \mathbf{R}\right)$, where the Dirac delta function $\delta\left(\mathbf{r}-\mathbf{r}^{\prime}: \mathbf{R}\right)$ applies to all irreducible representations of $\mathrm{S}_{n_{t}}$ spanned by the basis, whereas the closure expression is $\boldsymbol{\Phi}_{\mathrm{A}}(\mathbf{r}: \mathbf{R}) \cdot \boldsymbol{\Phi}_{\mathrm{A}}\left(\mathbf{r}^{\prime}: \mathbf{R}\right)^{\dagger}=Q \delta_{\mathrm{A}}\left(\mathbf{r}-\mathbf{r}^{\prime}: \mathbf{R}\right)$ for the nonorthogonal explicitly antisymmetric representation of eq 4 , where $Q=n_{t} ! /$ $\left(n_{1} ! n_{2} ! \cdots n_{N} !\right)$ is the redundancy of the explicitly antisymmetric basis of eq 4 in the closure limit. ${ }^{13}$

B. Hamiltonian Matrices in the Spectral-Product Representations. It is convenient in connection with evaluation of matrix elements to rewrite the Hamiltonian operator of eq 2 in a form suggested by the assignments of groups of electrons to atoms made in eq 3 in constructing spectral-product matrix representatives. Specifically, letting $\mathbf{i}(\mathbf{j})$ designate the electrons on atom $\alpha(\beta)$, the Hamiltonian operator of eq 2 is written

$$
\hat{H}(\mathbf{r}: \mathbf{R})=\sum_{\alpha=1}^{N} \hat{H}^{(\alpha)}(\boldsymbol{i})+\sum_{\alpha=1}^{N-1} \sum_{\beta=\alpha+1}^{N} \hat{V}^{(\alpha, \beta)}\left(\mathbf{i}: \mathbf{j}: \mathbf{R}_{\alpha \beta}\right)
$$

where

$$
\hat{H}^{(\alpha)}(\mathbf{i})=\sum_{i}^{n_{\alpha}}\left\{-\frac{\hbar^{2}}{2 m} \nabla_{i}^{2}-\frac{Z_{\alpha} e^{2}}{r_{i \alpha}}\right\}+\sum_{i}^{n_{\alpha}-1} \sum_{i^{\prime}=i+1}^{n_{\alpha}} \frac{e^{2}}{r_{i i^{\prime}}}
$$

includes the electronic terms arbitrarily associated with the atom $\alpha$, and

$$
\begin{array}{r}
\hat{V}^{(\alpha, \beta)}\left(\mathbf{i}: \mathbf{j}: \mathbf{R}_{\alpha \beta}\right)=\frac{Z_{\alpha} Z_{\beta} e^{2}}{R_{\alpha \beta}}-\sum_{i}^{n_{\alpha}} \frac{Z_{\beta} e^{2}}{r_{i \beta}}-\sum_{j}^{n_{\beta}} \frac{Z_{\alpha} e^{2}}{r_{j \alpha}}+ \\
\sum_{i}^{n_{\alpha}} \sum_{j}^{n_{\beta}} \frac{e^{2}}{r_{i j}}
\end{array}
$$

includes the Coulombic interaction terms between the pair of atoms $(\alpha, \beta)$.

Employing the basis sets of eqs 3 and 4 in variational solutions of eq 1 gives the matrix Schrödinger equations appropriate for each of the two representation

$$
\mathbf{H}(\mathbf{R}) \cdot \mathbf{U}_{\mathrm{H}}(\mathbf{R})=\mathbf{U}_{\mathrm{H}}(\mathbf{R}) \cdot \mathbf{E}(\mathbf{R})
$$

and

$$
\mathbf{H}_{\mathrm{A}}(\mathbf{R}) \cdot \mathbf{V}_{\mathrm{H}}(\mathbf{R})=\mathbf{S}(\mathbf{R}) \cdot \mathbf{V}_{\mathrm{H}}(\mathbf{R}) \cdot \mathbf{E}(\mathbf{R})
$$

where

$$
\begin{gathered}
\mathbf{H}(\mathbf{R}) \equiv\langle\boldsymbol{\Phi}(\mathbf{r}: \mathbf{R})|\hat{H}(\mathbf{r}: \mathbf{R})| \boldsymbol{\Phi}(\mathbf{r}: \mathbf{R})\rangle=\sum_{\alpha=1}^{N} \mathbf{H}^{(\alpha)}+ \\
\sum_{\alpha=1}^{N-1} \sum_{\beta=\alpha+1}^{N} \mathbf{V}^{(\alpha, \beta)}\left(\mathbf{R}_{\alpha \beta}\right)
\end{gathered}
$$

and

$$
\begin{array}{r}
\mathbf{H}_{\mathrm{A}}(\mathbf{R}) \equiv\left\langle\boldsymbol{\Phi}_{\mathrm{A}}(\mathbf{r}: \mathbf{R})|\hat{H}(\mathbf{r}: \mathbf{R})| \mathbf{\Phi}_{\mathrm{A}}(\mathbf{r}: \mathbf{R})\right\rangle=\sum_{\alpha=1}^{N} \mathbf{H}^{(\alpha)}(\mathbf{R})+ \\
\sum_{\alpha=1}^{N-1} \sum_{\beta=\alpha+1}^{N} \mathbf{V}^{(\alpha, \beta)}(\mathbf{R})
\end{array}
$$

are the respective Hamiltonian matrices. Whereas eqs $6-8$ have been employed in obtaining eqs 9 and 11, since the groups of electrons in eqs 7 and 8 share the assignments of eq 3 , it is necessary to use the defining Hamiltonian operator of eq 2 in obtaining eqs 10 and 12 for the representation of eq 4, in which case prior antisymmetry prohibits such assignments of electrons to particular atoms.

In eq 9, the unitary solution matrix $\mathbf{U}_{\mathrm{H}}(\mathbf{R})$ diagonalizes the Hamiltonian matrix of eq 11 constructed in the orthonormal basis of eq 3. In contrast, the matrix $\mathbf{V}_{\mathrm{H}}(\mathbf{R})$ in eq 10 is generally not unitary since the metrically defined Hamiltonian matrix of eq 12 is constructed in the nonorthogonal basis of eq 4 . Rather, $\mathbf{V}_{\mathrm{H}}(\mathbf{R})$ satisfies the familiar condition $\mathbf{V}_{\mathrm{H}}(\mathbf{R})^{\dagger} \cdot \mathbf{S}(\mathbf{R}) \cdot \mathbf{V}_{\mathrm{H}}(\mathbf{R})=$ $\mathbf{I},{ }^{17}$ with the metric matrix $\mathbf{S}(\mathbf{R})$ here and in eq 10 given by the expression

$$
\mathbf{S}(\mathbf{R}) \equiv\left\langle\hat{P}_{\mathrm{A}} \boldsymbol{\Phi}(\mathbf{r}: \mathbf{R}) \mid \hat{P}_{\mathrm{A}} \boldsymbol{\Phi}(\mathbf{r}: \mathbf{R})\right\rangle=Q^{1 / 2}\left\langle\boldsymbol{\Phi}(\mathbf{r}: \mathbf{R})\left|\hat{P}_{\mathrm{A}}\right| \boldsymbol{\Phi}(\mathbf{r}: \mathbf{R})\right\rangle
$$

where the factor $Q=n_{t} ! /\left(n_{1} ! n_{2} ! \cdots n_{N} !\right)$ is the aforementioned redundancy of the explicitly antisymmetric basis of eq 4 in the closure limit. ${ }^{13}$

Evidently, $\mathbf{S}(\mathbf{R})$ of eq 13 has an interpretation as the matrix representative of the antisymmetrizer of eq 5 in the basis of eq 3 , separate from its role as the metric matrix of the nonorthogonal basis of eq 4. Accordingly, since the antisymmetrizer can be normalized as an idempotent or projector operator having eigenvalues 0 and 1 only, ${ }^{23}$ it is seen that the allowable eigenvalues of the $\mathbf{S}(\mathbf{R})$ matrix of eq 13 must converge to 0 and $Q$ in the closure limit, with the value $Q$ appearing in place of 1 consequent of the wave function normalization convention adopted in eq 5 .

Although the Hamiltonian matrices of eqs 11 and 12 are similar in form, the individual atomic and atomic-pairwise interaction terms shown there differ significantly in the two representations. Specifically, the atomic terms in eq 11 obtained from eq 7 are independent of atomic position, and the Coulombic interaction terms obtained from eq 8 depend only on the vector separation $\mathbf{R}_{\alpha \beta}$ of an individual atomic pair $(\alpha, \beta)$. In contrast, the terms in eq 12, which must be evaluated using the Hamiltonian of eq 2, formally depend on the positions $\mathbf{R}$ of all the atoms in the aggregate due to the overall electron antisymmetry of the basis of eq 4 . That is, because the electronic 
coordinates in the representation of eq 4 appear at all the atomic centers, the individual Coulombic interactions in the Hamiltonian of eq 2 can be alternatively inter- or intra-atomic terms, and the partitioning of eqs $6-8$, which constitutes an assignment of particular electrons to specific atomic centers, can not be employed in this case.

The simpler forms of the terms appearing in eq 11 relative to those in eq 12 are consequences of the orthonormality of the atomic spectral-product basis employed in the absence of prior enforcement of overall aggregate electron antisymmetry, the use of atomic eigenstates in the representation, and the atomic pairwise-additive nature of the interaction terms in the Hamiltonian operator of eq 6 . In contrast, the atomic-pairwise nature of the interaction terms in the Hamiltonian operator can not be employed to advantage in conjunction with use of the explicitly antisymmetric representation of eq 4 . It spite of the apparent differences in the two representations, both can give the same physical Schrödinger eigenstates and energies in appropriate closure limits, as described below.

C. Isolation of Physical Eigenstates in the SpectralProduct Representations. It has been shown that the basis of eq 3 spans the totally antisymmetric representation of the aggregate $n_{t}$-electron symmetric group $\mathrm{S}_{n_{t}}$ once and only once in the limit of closure, although other unphysical non totally antisymmetric (non-Pauli) representations of $\mathrm{S}_{n_{t}}$ are also spanned by the basis. ${ }^{13}$ The non-Pauli solutions are accordingly included in the columns of the unitary transformation matrix $\mathbf{U}_{\mathrm{H}}(\mathbf{R})$ obtained from solution of eq 9. In contrast, although the solutions of eq 10 are totally antisymmetric by construction, they generally become numerically unstable in large representations, consequent of the linear dependence arising from the $Q$-fold redundancy of the basis of eq 4 in the closure limit.

The desired Pauli and linearly independent solutions can be obtained by transforming eqs 9 and 10 to representations which isolate totally antisymmetric and linearly independent subspaces, respectively. The unitary transformation matrix $\mathbf{U}_{\mathrm{S}}(\mathbf{R})$ required to accomplish this partitioning of eqs 9 and 10 is obtained from diagonalization

$$
\begin{aligned}
& \mathbf{U}_{\mathrm{S}}(\mathbf{R})^{\dagger} \cdot \mathbf{S}(\mathbf{R}) \cdot \mathbf{U}_{\mathrm{S}}(\mathbf{R})=\mathbf{s}_{\mathrm{d}}(\mathbf{R})= \\
&\left(\begin{array}{ll}
\left\{\mathbf{s}_{\mathrm{d}}(\mathbf{R})\right\}_{p p} & \mathbf{0} \\
\mathbf{0} & \left\{\mathbf{s}_{\mathrm{d}}(\mathbf{R})\right\}_{u u}
\end{array}\right) \rightarrow\left(\begin{array}{ll}
Q \mathbf{I}_{p p} & \mathbf{0} \\
\mathbf{0} & \mathbf{0}
\end{array}\right)
\end{aligned}
$$

of the Hermitian metric matrix of eq $13 .{ }^{12,13}$ Here, the nonnegative eigenvalue matrix $\mathbf{S}_{\mathrm{d}}(\mathbf{R})$ is partitioned into an upper diagonal $\left\{\mathbf{s}_{\mathrm{d}}(\mathbf{R})\right\}_{p p}$ containing the largest eigenvalues, which tend to the indicated common redundancy factor $Q$ in the closure limit, and a lower diagonal $\left\{\mathbf{S}_{\mathrm{d}}(\mathbf{R})\right\}_{u u}$ of smaller eigenvalues which tend to zero in the limit. This ordering is obtained in the usual way by appropriate arrangement of the columns of the transformation matrix $\mathbf{U}_{\mathrm{S}}(\mathbf{R})$ into physical $(p)$ blocks on the lefthand side of the transformation matrix and unphysical $(u)$ blocks on the right-hand side.

The diagonalization of eq 14 is seen to correspond to construction of eigenstates of the Hermitian antisymmetrizer in the spectral-product basis of eq 3 in the form ${ }^{13}$

$$
\boldsymbol{\Phi}_{\mathrm{S}}(\mathbf{r}: \mathbf{R}) \equiv \boldsymbol{\Phi}(\mathbf{r}: \mathbf{R}) \cdot \mathbf{U}_{\mathrm{S}}(\mathbf{R}) \rightarrow\left\{\left\{\boldsymbol{\Phi}_{\mathrm{S}}(\mathbf{r}: \mathbf{R})\right\}_{p},\left\{\boldsymbol{\Phi}_{\mathrm{S}}(\mathbf{r}: \mathbf{R})\right\}_{u}\right\}
$$

where $\left\{\boldsymbol{\Phi}_{\mathrm{S}}(\mathbf{r}: \mathbf{R})\right\}_{p}$ contains totally antisymmetric states corresponding to the nonzero eigenvalues of the antisymmetrizer, and $\left\{\boldsymbol{\Phi}_{\mathrm{S}}(\mathbf{r}: \mathbf{R})\right\}_{u}$ contains non-Pauli states corresponding to the zero eigenvalues of the antisymmetrizer in the closure limit. In contrast, in the antisymmetric basis of eq 4 , the states generated by the transformation matrix $\mathbf{U}_{\mathrm{S}}(\mathbf{R})$ of eq 14 are written in the familiar canonically orthogonalized form ${ }^{24}$

$$
\begin{aligned}
& \boldsymbol{\Phi}_{\mathrm{S}}(\mathbf{r}: \mathbf{R}) \equiv \boldsymbol{\Phi}_{\mathrm{A}}(\mathbf{r}: \mathbf{R}) \cdot \mathbf{U}_{\mathrm{S}}(\mathbf{R}) \cdot \mathbf{s}_{\mathrm{d}}(\mathbf{R})^{1 / 2} \rightarrow \\
&\left\{\left\{\boldsymbol{\Phi}_{\mathrm{S}}(\mathbf{r}: \mathbf{R})\right\}_{p},\left\{\boldsymbol{\Phi}_{\mathrm{S}}(\mathbf{r}: \mathbf{R})\right\}_{u} \rightarrow 0\right\}
\end{aligned}
$$

These states include correctly normalized linearly independent combinations $(p)$ of the original basis, as well as un-normalizable null states associated with linearly dependent combinations $(u)$ of the basis of eq 4. Of course, in the case of degenerate eigenvalues, the eigenstates of eqs 15 and 16 are generally arbitrary but are nevertheless separated by the development into two invariant subspaces. In the large-separation limit $\mathbf{S}(\mathbf{R} \rightarrow \infty)$ $\rightarrow \mathbf{I}$, in which case the eigenvalues of the metric matrix are all unity, both representations of eqs 3 and 4 are orthonormal, and the states of eqs 15 and 16 are not required.

Equations 14 and 15 indicate that the eigenvalue spectrum of the matrix $\mathbf{S}(\mathbf{R})$ in any finite representation will fall in the finite interval $(0, Q)$, with the upper $(Q)$ and lower (0) limiting points corresponding to the correctly converged eigenvalues $Q$ and 0 of the antisymmetrizer $P_{\mathrm{A}}$ of eq $5 .^{23}$ The corresponding eigenvectors in finite representations will generally be mixtures of totally antisymmetric and non totally antisymmetric states. As the representation is enlarged, the eigenvalues will converge to the upper and lower limiting points, and the corresponding eigenvectors in the groupings of eq 15 will converge to the physically significant totally antisymmetric space $P_{\mathrm{A}}\left\{\boldsymbol{\Phi}_{\mathbf{S}}(\mathbf{r}: \mathbf{R})\right\}_{p}$ $\rightarrow Q^{1 / 2}\left\{\boldsymbol{\Phi}_{\mathbf{S}}(\mathbf{r}: \mathbf{R})\right\}_{p}$, and to the non-Pauli null space $P_{\mathrm{A}}\left\{\boldsymbol{\Phi}_{\mathbf{S}}(\mathbf{r}:\right.$ $\mathbf{R})\}_{u} \rightarrow \mathbf{0} .{ }^{13}$ The linearly independent combinations of the termby-term antisymmetric basis functions of eq 4 given by eq 16 become identical to the totally antisymmetric states of eq 15 in the closure limit, ${ }^{13}$ whereas the linearly dependent states of eq 16 are seen to be eliminated by the development in the standard way. ${ }^{24}$

The physically significant blocks of the Hamiltonian matrices of eqs 11 and 12 are obtained by transforming from the $\boldsymbol{\Phi}(\mathbf{r}$ : $\mathbf{R})$ and $\boldsymbol{\Phi}_{\mathrm{A}}(\mathbf{r}: \mathbf{R})$ representations of eqs 3 and 4, respectively, to the physically invariant subspaces $\boldsymbol{\Phi}_{\mathrm{S}}(\mathbf{r}: \mathbf{R})$ given by eqs 15 and 16. This is accomplished in the forms ${ }^{13}$

$$
\left\{\mathbf{H}_{\mathrm{A}}(\mathbf{R})\right\}_{p p} \equiv\left\{\mathbf{U}_{\mathrm{S}}(\mathbf{R})^{\dagger} \cdot \mathbf{H}(\mathbf{R}) \cdot \mathbf{U}_{\mathrm{S}}(\mathbf{R})\right\}_{p p}
$$

$$
\begin{aligned}
& \left\{\mathbf{H}_{\mathrm{A}}(\mathbf{R})\right\}_{p p} \equiv \\
& \quad\left\{\mathbf{S}_{\mathrm{d}}(\mathbf{R})^{-1 / 2}\right\}_{p p} \cdot\left\{\mathbf{U}_{\mathrm{S}}(\mathbf{R})^{\dagger} \cdot \mathbf{H}_{\mathrm{A}}(\mathbf{R}) \cdot \mathbf{U}_{\mathrm{S}}(\mathbf{R})\right\}_{p p} \cdot\left\{\mathbf{s}_{\mathrm{d}}(\mathbf{R})^{-1 / 2}\right\}_{p p}
\end{aligned}
$$

employing the matrix $\mathbf{U}_{\mathrm{S}}(\mathbf{R})$, where the subscript ( $p p$ ) refers to the upper left-hand blocks of the enclosed matrices (cf., eqs 14-16). The unitary transformation matrix $\mathbf{U}_{\mathrm{S}}(\mathbf{R})$ of eq 14 obtained from the metric matrix of eq 13 is therefore seen to be responsible for incorporating the nonlocal effects of interatomic aggregate electron permutation symmetry in the Hamiltonian matrix of eq 11 by virtue of eq 17 . As a consequence, all terms in both Hamiltonian matrices now depend formally on the positions $\mathbf{R}$ of all the atoms in the aggregate.

Overall electron antisymmetry can be enforced as required in this approach in accordance with the spatial separations of the individual atoms and the associated perceived strengths of 
their interactions in the Hamiltonian of eq $17,{ }^{14}$ rather than as a possibly encumbering overall global constraint, as employed in the more conventional Hamiltonian matrix in eq $18 .{ }^{8}$ Similarly, the role of the transformation matrix $\mathbf{U}_{\mathrm{S}}(\mathbf{R})$ in the explicitly antisymmetric representation of eq 4 is to isolate its linearly independent subspace, in which connection the spatial proximity of the constituents atoms clearly plays a central role, emphasizing the underlying identical purposes of eqs 17 and 18 in isolating a common invariant subspace in which to express the Hamiltonian matrix.

Finally, identical physically significant Schrödinger eigenstates are obtained from the immediately foregoing Hamiltonian matrices in the form $\boldsymbol{\Psi}_{p}(\mathbf{r}: \mathbf{R})=\{\boldsymbol{\Phi}(\mathbf{r}: \mathbf{R})\}_{p} \cdot\left\{\mathbf{U}_{\mathbf{H}}(\mathbf{R})\right\}_{p p}$, where $\{\boldsymbol{\Phi}(\mathbf{r}: \mathbf{R})\}_{p}$ is obtained from either eq 15 or eq 16 and $\left\{\mathbf{U}_{\mathrm{H}}(\mathbf{R})\right\}_{p p}$ is obtained from either of the Hamiltonian matrices of eqs 17 or 18. Although the requirements of computational implementations of the two approaches differ significantly, in view of the "post" and "prior" incorporation of aggregate electron antisymmetry in the two Hamiltonian matrices, the developments are clearly united through use of the metric matrix of eq 13 in isolating a common invariant physical subspace in which to construct Schrödinger eigensolutions.

\section{Computational Implementations and Applications}

Algorithms and computer codes have been devised to evaluate the various expressions given in the preceding sections. In brief, a valence-bond code suite $\left(\mathrm{CRUNCH}^{25}\right)$ has been modified to accommodate the use of real Slater orbitals in evaluating both atomic and molecular integrals $\left(\right.$ SMILES $\left.^{26}\right)$ for performing configuration-interaction calculations of atomic eigenstates and other information required to implement the spectral-product development. Calculations are performed with this code suite combination employing standard valence-bond tableau descriptions of configurational-state functions, ${ }^{6,26}$ and of the Hamiltonian and metric matrix elements, appropriate for both atomic eigenstates and their atomic-pair interactions. The use of common atomic orbitals and configurational-state functions for both atomic eigenstates and their pairwise interactions, performed as diatomic calculations, facilitates the transformation from valence-bond wave functions and matrix elements to the corresponding quantities in the spectral-product formalism.

Specifically, the transformation from atomic valence-bond configurational-state functions $\boldsymbol{\Phi}_{v b}^{(\alpha)}(\mathbf{i})$ to atomic eigenfunctions $\boldsymbol{\Phi}^{(\alpha)}(\mathbf{i})$ takes the form $\boldsymbol{\Phi}^{(\alpha)}(\mathbf{i})=\boldsymbol{\Phi}_{v b}^{(\alpha)}(\mathbf{i}) \cdot \mathbf{V}^{(\alpha)}$, where the matrix $\mathbf{V}^{(\alpha)}$ and the associated atomic energies are obtained directly from the valence-bond configuration-interaction calculation for the atom $\alpha$. This information for a pair of atoms $\alpha$ and $\beta$ provides the transformation matrix $\mathbf{V}^{(\alpha, \beta)}=\left\{\mathbf{V}^{(\alpha)} \otimes \mathbf{V}^{(\beta)}\right\}_{\mathrm{O}}$ from the valence-bond configurational-state functions for the $\alpha, \beta$ pair to the corresponding spectral-product functions, where the subscript " $\mathrm{O}$ " refers to the product-ordering convention of eq 3. The transformation matrix $\mathbf{V}^{(\alpha, \beta)}$ is sufficient for determination of the pairwise-interaction energies required in eqs 11 and 12, whereas the additional ordered-product transformation matrix of the form $\left\{\mathbf{V}^{(\alpha)} \otimes \mathbf{V}^{(\beta)} \otimes \mathbf{V}^{(\gamma)}\right\}_{\mathrm{O}}$ is required for determination of the metric matrix of eq 13 for the triatomic systems of interest here.

Computational results obtained in this way are reported in section $\mathrm{A}$ for $\mathrm{H}_{2}$ and $\mathrm{H}_{3}$ molecules, in section $\mathrm{B}$ for diatomic $\mathrm{CH}$, and in section $\mathrm{C}$ for triatomic $\mathrm{CH}_{2}$. These calculations are illustrative of selected aspects of the spectral-product approach, and of the capabilities of the computational algorithms and codes devised, and are not meant to provide exhaustive computational descriptions of the quantities reported.

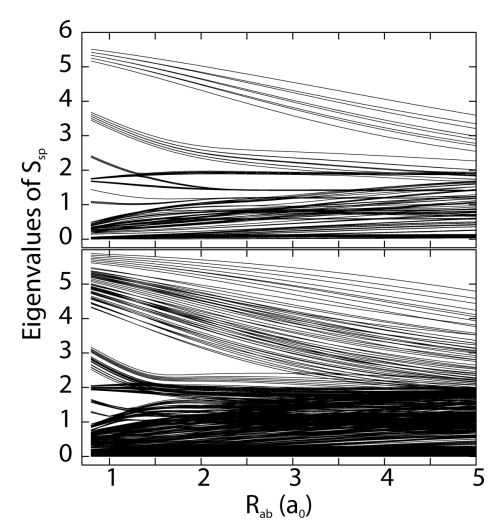

Figure 1. Eigenvalues of the doublet- and quartet-state metric matrix of eq 13 for symmetric collinear $\mathrm{H}_{3}\left(\mathrm{H}_{a}-\mathrm{H}_{b}-\mathrm{H}_{c}\right)$ as functions of atomic separation $\left(R_{a b}=R_{b c}\right)$ : upper panel, $2 \mathrm{~s} 1 \mathrm{p}$ atomic basis (375 terms); lower panel, $3 \mathrm{~s} 2 \mathrm{p}$ atomic basis (2187 terms). The limiting points of the spectra are 0 and $Q=3 ! /(1 ! 1 ! 1 !)=6$, the latter value specifying the redundancy of the antisymmetric basis of eq 4 in the closure limit.

A. Diatomic and Triatomic Hydrogen Molecules. Although the metric matrix is familiar in connection with canonical transformations of one-electron orbital basis sets for Hartree-Fock calculations, ${ }^{24}$ its attributes and importance in connection with many-electron basis states are perhaps less familiar, particularly in the context of the spectral-product representations described here. Of particular interest is the role of the metric matrix in providing a measure of the spectral closure of the representation of eq 3 in the absence of explicit antisymmetry, and of the redundancy of the representation of eq 4, particularly in connection with commonly employed ionic terms involving the transfer of one or more electrons from one atom to others. Illustrative molecular calculations involving hydrogen atoms help to clarify these issues, and also demonstrate the capabilities of the algorithms and codes devised in these familiar cases.

In Figure 1 are shown as an example the eigenvalues of the metric matrix of eq 13 for symmetric collinear $\mathrm{H}_{3}$ as a function of atomic separation $\left(R_{a b}=R_{b c}\right)$, constructed in $2 \mathrm{~s} 1 \mathrm{p}$ and $3 \mathrm{~s} 2 \mathrm{p}$ atomic Slater basis sets employing hydrogenic exponents. These atomic orbital basis sets give doublet spectral-product representations of dimensions 375 (upper panel) and 2187 (lower panel) of the forms $\boldsymbol{\Phi}^{\left(\mathrm{H}_{3}\right)}(\mathbf{r}: \mathbf{R})=\left\{\boldsymbol{\Phi}^{\left(\mathrm{H}_{a}\right)}(\mathbf{1}) \otimes \boldsymbol{\Phi}^{\left(\mathrm{H}_{b}\right)}(\mathbf{2}) \otimes \boldsymbol{\Phi}^{\left(\mathrm{H}_{c}\right)}(\mathbf{3})\right\}_{\mathrm{O}}$ of eq 3 . The eigenvalues of the antisymmetrizer of eq 5 in these representations are seen to appear only in the allowable interval $(0, Q)$ where $Q=3 ! /(1 ! 1 ! 1 !)=6$, and to largely span the interval for smaller values of atomic separation. The great many values near 0 in the lower panel are in accordance with the lower end of the spectrum of the projector $\hat{P}_{\mathrm{A}}$ providing a point of accumulation. ${ }^{23}$ The eigenstates corresponding to these approximately zero eigenvalues refer to non totally antisymmetric states in the product basis (eqs 3 and 15), whereas in the prior antisymmetrized basis they refer to linearly dependent states (eqs 4 and 16). Conversely, the states of eqs 15 and 16 corresponding to the largest eigenvalues of Figure 1 refer to approximately totally antisymmetric and linearly independent states, respectively. Note that the value $Q=6$ implies the antisymmetric basis of eq 4 is 6-fold redundant in the limit of closure in this case, the redundancy of which is removed by the canonical orthogonalization procedure of eq 16. As the number of basis functions and their spatial extent is increased, the eigenvalues of Figure 1 converge to the upper and lower limiting points of the spectrum for finite values of atomic separation, whereas they approach unity in the limit of arbitrarily large separation in any square-integrable representation. 


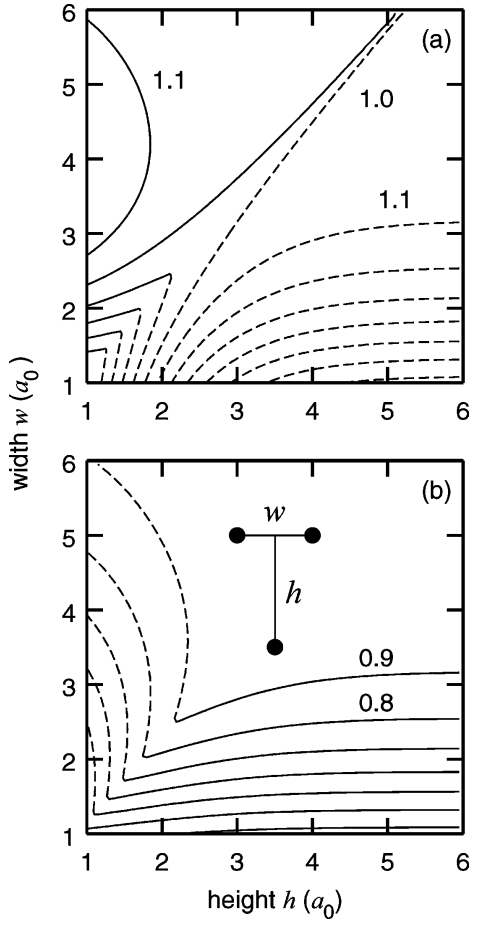

Figure 2. Constant-value contours for the two doublet eigenvalue surfaces of the metric matrix of eq 13 for T-shaped arrangements in $\mathrm{H}_{3}$ constructed in a minimal $1 \mathrm{~s}^{3}$ representation, employing increments/ decrements of 0.1 between adjacent contours in each case: (a) upper surface; (b) lower surface. The two surfaces join along the line $w=$ $1.155 h$ line, corresponding to $D_{3 h}$ symmetry, ${ }^{27}$ with the solid and dashed lines indicating the continuity of surface crossings along this line.

Although large orbital basis sets are generally required to achieve convergence in spectral-product representations, ${ }^{13}$ metric matrices constructed even in small basis sets provide useful information. ${ }^{14}$ In Figure 2 are shown constant-value contours for the two doublet eigenvalues of the metric matrix obtained in a minimal $1 \mathrm{~s}^{3}$ spectral-product representation for T-shaped $\mathrm{H}_{3}$ as functions of the width $(w)$ and height $(h)$ of the T. In (a), the contours that depict the higher-lying surface are monotonically increasing from that labeled 1.0 , whereas in (b) depicting the lower surface, they are monotonically decreasing from that labeled 0.9. The two surfaces have downward and upward facing cusps, respectively, in their contours along a seam of intersection following the line $w=1.155 h$, which corresponds to a $D_{3 h}$ highsymmetry arrangement of the three atoms.

The high-symmetry seam in Figure 2 anticipates the betterknown $D_{3 h}$ seam of intersection of the two lowest-lying doublet energy surfaces in $\mathrm{H}_{3},{ }^{27}$ this feature identified here entirely on basis of calculations of the eigenvalues of the metric matrix. Such calculations of metric matrices, which entail evaluations only of overlap matrix elements, can provide useful information in other molecular cases more generally in the absence of any energy calculations whatsoever.

The spectral-product representation of eq 3 is complete, employing only the indicated neutral atomic states, ${ }^{13}$ and the associated antisymmetrized basis of eq 4 is correspondingly $Q$-fold redundant in the closure limit. It is instructive in this connection to examine the effects of adding commonly employed ionic or charge-transfer configurations to the atomic spectral-product basis of eq 3 , and to demonstrate quantitatively the additional redundancy added thereby. In Figure 3 are shown the singlet and triplet eigenvalues of the metric matrix of eq 13 for diatomic hydrogen constructed in a 7s5p3d2f1g Slater basis set employing fixed hydrogen $1 \mathrm{~s}, 2 \mathrm{p}, 3 \mathrm{~d}, 4 \mathrm{f}, 5 \mathrm{~g}$ exponents in

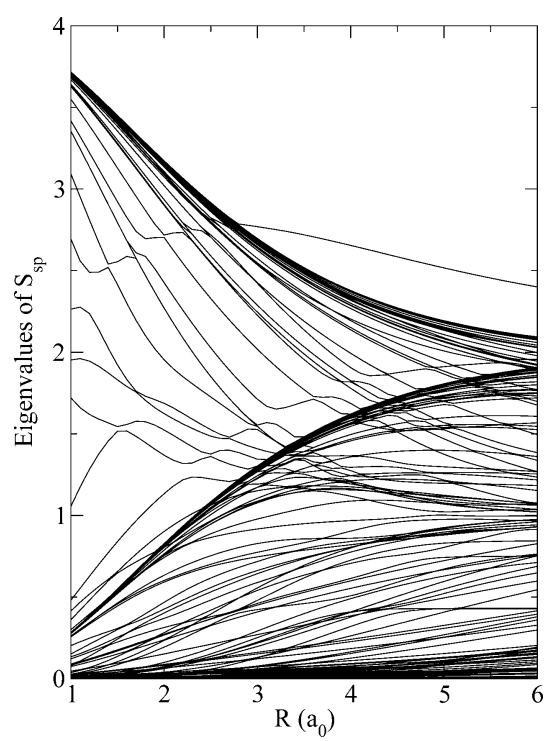

Figure 3. Eigenvalues of the singlet- and triplet-state metric matrix of eq 13 for diatomic hydrogen $\mathrm{H}_{2}$ constructed in a $7 \mathrm{~s} 5 \mathrm{p} 3 \mathrm{~d} 2 \mathrm{f} 1 \mathrm{~g}$ Sturmian Slater basis set, ${ }^{28,29}$ including both covalent (119) and chargetransfer (154) valence-bond excitations from the Heitler-London configurations. The origins of the structures evident in the plot, and of the spectral interval $(0,4)$ in this case, are discussed in the text.

forming Sturmian sequences. ${ }^{28}$ The eigenvalues shown correspond to calculations which include all single-excitations from the Heitler-London configuration, including both covalent $\left(\mathrm{H}-\mathrm{H}, 119\right.$ terms) and ionic $\left(\mathrm{H}^{+} \mathrm{H}^{-}\right.$and $\mathrm{H}^{-} \mathrm{H}^{+}, 154$ terms $)$ structures. Although the redundancy factor is $Q=2 ! /(1 ! 1 !)=$ 2 for the antisymmetrized spectral-product or covalent representations of eq 4 , the presence of the additional charge-transfer terms in Figure 3 is seen to give rise to eigenvalues which appear in the larger interval $(0,4)$ in this case. This larger interval is a consequence of the 4-fold redundancy in the closure limit of the combined covalent and ionic basis employed in the calculations of Figure 3, whereas the band of near zero eigenvalues in Figure 3 arises from the linear-dependence of charge-transfer terms with the diffuse covalent excitations; this band is absent in the eigenvalue spectrum $(0,2)$ obtained with covalent terms alone employing the same orbital basis set. Moreover, the bands of eigenvalues approaching 2 for larger separation are also consequences of the charge-transfer terms, which continue to provide a 2 -fold redundant explicitly antisymmetric representation in the dissociation limit, whereas the bands of eigenvalues approaching unity at large separation arise from the covalent structures in the representation.

The results of Figure 3 indicate the eigenvalues of the metric matrix can form a complex pattern that can distinguish among the types of structures present in the representation employed, largely confirming the redundancy of covalent and ionic terms commonly employed in molecular calculations, and emphasizing the importance of removing these redundancies in explicitly antisymmetrized large-basis-set calculations by the canonical orthogonalization of eq 18.

The capabilities of the Slater-based spectral-product codes devised for energy calculations are illustrated in Figures 4 and 5 , which depict low-lying $(n=1)$ and excited $(n=2)$ singlet and triplet potential energy curves, respectively, in $\mathrm{H}_{2}$ obtained from full configuration-interaction calculations in an optimal $5 \mathrm{~s} 3 \mathrm{p} 2 \mathrm{~d} 1 \mathrm{f}$ valence Slater basis. ${ }^{29}$ These results are in excellent agreement with the accepted ground-state $(n=1)$ values,${ }^{30}$ and are in generally good but not precise accord with the most 


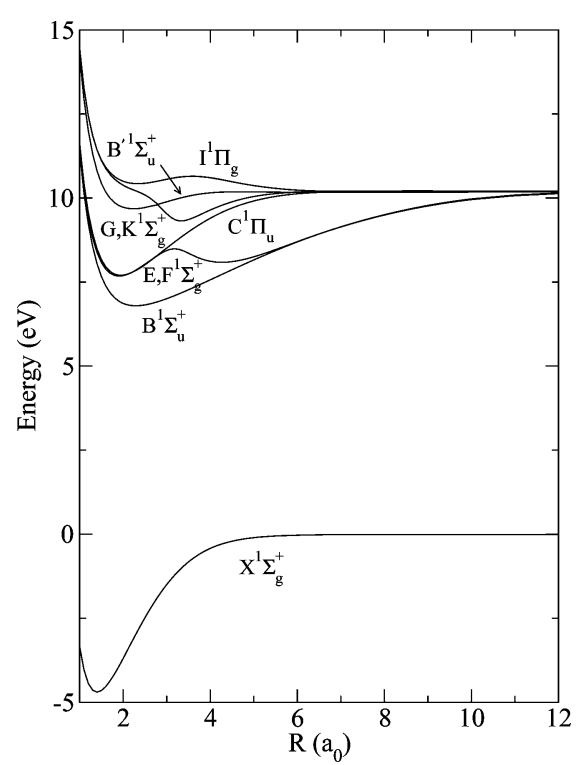

Figure 4. Singlet-state potential energy curves dissociating to $n=1$ and 2 limits in diatomic hydrogen $\mathrm{H}_{2}$, obtained from full (1953 terms) configuration-interaction calculations which include both covalent and charge-transfer terms in an optimized $5 \mathrm{~s} 3 \mathrm{p} 2 \mathrm{~d} 1 \mathrm{f}$ valence basis of Slater orbitals, ${ }^{29}$ employing computer codes devised recently for this purpose.

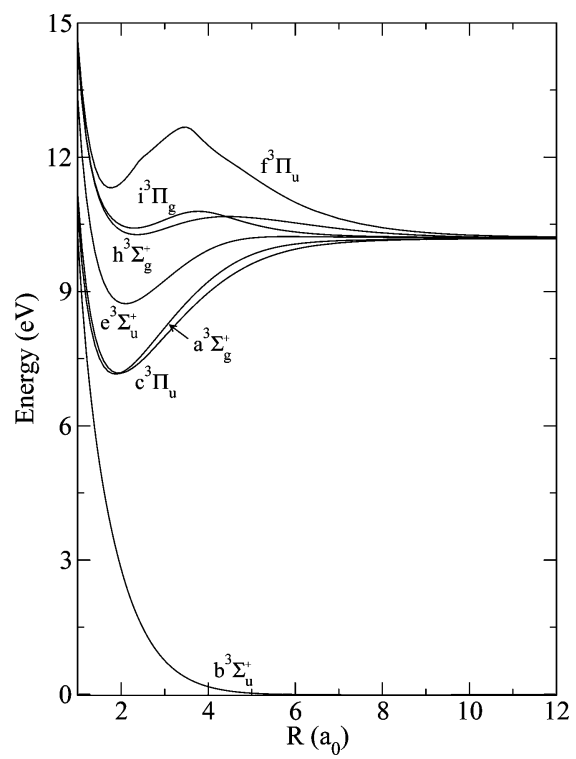

Figure 5. Triplet-state potential energy curves dissociating to $n=1$ and 2 limits in diatomic hydrogen $\mathrm{H}_{2}$, obtained from full (1891 terms) configuration-interaction calculations which include both covalent and charge-transfer terms in an optimized $5 \mathrm{~s} 3 \mathrm{p} 2 \mathrm{~d} 1 \mathrm{f}$ valence basis of Slater orbitals, ${ }^{29}$ employing computer codes devised recently for this purpose.

accurate previous calculations available for the indicated excited $(n=2)$ states. $^{30}$

Of particular interest in Figure 4 are the shapes of the $\mathrm{B}^{1} \Sigma_{\mathrm{u}}^{+}$ and $\mathrm{E}, \mathrm{F}^{1} \Sigma_{\mathrm{g}}^{+}$curves, and the presence of a double well in the latter curve and in the $\mathrm{G}, \mathrm{K}^{1} \Sigma_{\mathrm{g}}^{+}$curve, and the large barrier in the $\mathrm{f}^{3} \Pi_{\mathrm{u}}$ state in Figure 5. The long-range nature of the calculated $\mathrm{B}^{1} \sum_{\mathrm{u}}^{+}$and $\mathrm{E}, \mathrm{F}^{1} \Sigma_{\mathrm{g}}^{+}$curves, which provide good approximations to the accurate values ${ }^{30}$ is commonly attributed to contributions from charge-transfer terms, whereas the calculated $\mathrm{G}, \mathrm{K}^{1} \Sigma_{\mathrm{g}}^{+}$and $\mathrm{f}^{3} \Pi_{\mathrm{u}}$ curves provide reasonably quantitative descriptions of the known detailed shapes of these two curves but are not in precise agreement with the results of highly accurate calculations. ${ }^{30}$ Of course, the high-lying charge-transfer structure $\left(\mathrm{H}^{+}+\mathrm{H}^{-}\right)$is stable and gives rise to a diabatic potential curve that contributes significantly to the $\mathrm{B}^{1} \Sigma_{\mathrm{u}}^{+}$and $\mathrm{E}, \mathrm{F}^{1} \Sigma_{\mathrm{g}}^{+}$curves of Figure 4, but this circumstance does not invalidate the foregoing assertions that charge-transfer structures are already present in the spectral-product description of eq 3 in the closure limit, as the results of Figure 3 indicate.

Highly accurate calculation of the excited-state potential energy curves of $\mathrm{H}_{2}$ are generally performed by employing special basis sets on a state-by-state basis. It is therefore satisfying that the results of Figures 4 and 5 are obtained from single diagonalizations of Hamiltonian matrices constructed in Slater-based spectral-product representations employing the algorithms and codes devised, which are applicable to other molecules more generally.

B. Diatomic CH Molecule. The foregoing spectral-product representations of molecules containing hydrogen atoms $\left(\mathrm{H}_{2}\right.$, $\mathrm{H}_{3}$ ) are closely related to corresponding valence-bond descriptions, the two approaches differing largely in the normalization conventions employed for orbital-product states, and a prediagonalization of the atomic hydrogen Hamiltonian employed in the spectral-product development. The situation is quite different for molecules containing many-electron atoms. The differences between metric and Hamiltonian matrices constructed in valence-bond and spectral-product representation can be illustrated in many-electron-atom cases employing the wellstudied diatomic $\mathrm{CH}$ molecule as a simple example. ${ }^{15}$ In this example, both valence-bond and spectral-product metric and Hamiltonian matrices are constructed by employing identical hydrogen atom $1 \mathrm{~s}$ spin orbitals and $1 \mathrm{~s}^{2}\left(2 \mathrm{~s}^{2} 2 \mathrm{p}^{2}+2 \mathrm{~s} 2 \mathrm{p}^{3}+2 \mathrm{p}^{4}\right)$ carbon atom valence-shell configurations, the latter giving rise to 55 distinct atomic multiplet states. The valence-bond states made from these orbital configurations are in the forms of standard tableau functions which are employed directly in constructing the required matrices, ${ }^{6,25}$ whereas in the spectralproduct representation the two hydrogen atom $\left[{ }^{2} \mathrm{~S}^{\mathrm{e}}\right]$ and 55 carbon atom $\left[(2)^{3} \mathrm{P}^{\mathrm{e}},(2)^{1} \mathrm{D}^{\mathrm{e}},(2){ }^{1} \mathrm{~S}^{\mathrm{e}},{ }^{5} \mathrm{~S}^{\mathrm{o}},{ }^{3} \mathrm{D}^{\mathrm{o}},{ }^{3} \mathrm{P}^{\mathrm{o}},{ }^{1} \mathrm{D}^{\mathrm{o}},{ }^{3} \mathrm{~S}^{\mathrm{o}},{ }^{1} \mathrm{P}^{\mathrm{o}}\right]$ multiplet states are constructed and the spectral-product matrices of eqs $11-13$ of dimension $2 \times 55=110$ are evaluated employing the aforementioned valence-bond $\rightarrow$ spectral-product transformation devised explicitly for this purpose.

In Figure 6 are shown eigenvalues of the doublet- and quartetstate metric matrix for diatomic $\mathrm{CH}$ constructed in the aforementioned atomic valence configurations using an optimized valence $(7 \mathrm{~s} 5 \mathrm{p} 3 \mathrm{~d} 2 \mathrm{f} 1 \mathrm{~g})$ atomic carbon orbital basis. ${ }^{29}$ The solid lines refer to eigenvalues of the metric matrix in the orthonormal spectral-product representation of eq 3 , whereas the dashed lines are those obtained in the same configurational basis employing the standard tableau functions of valence-bond theory. ${ }^{6,25}$ The two sets of eigenvalues of Figure 6 are evidently quite different, those in the valence-bond description grouping in accordance with the normalizations of standard tableau functions transforming under particular irreducible representations of $\mathrm{S}_{n_{t}}{ }^{19}$ and approaching different limits at large atomic separation. In contrast, there are only three distinct highly degenerate eigenvalues of the spectral-product metric matrix in Figure 6, which are seen to approach unity, in accordance with the orthonormality of the spectral-product representation at large atomic separation. The small number of distinct eigenvalues of the latter representation relates to use of only $n=1$ shell hydrogen and $n=2$ shell carbon multiplet configurations in the calculations, and to the orthogonality of the 110-term representation. Relatedly, although the allowable eigenvalue interval for $\mathrm{CH}$ formally extends to $Q=7 ! /(6 ! 1 ! 1 !)=7$, the 110 -term representation 


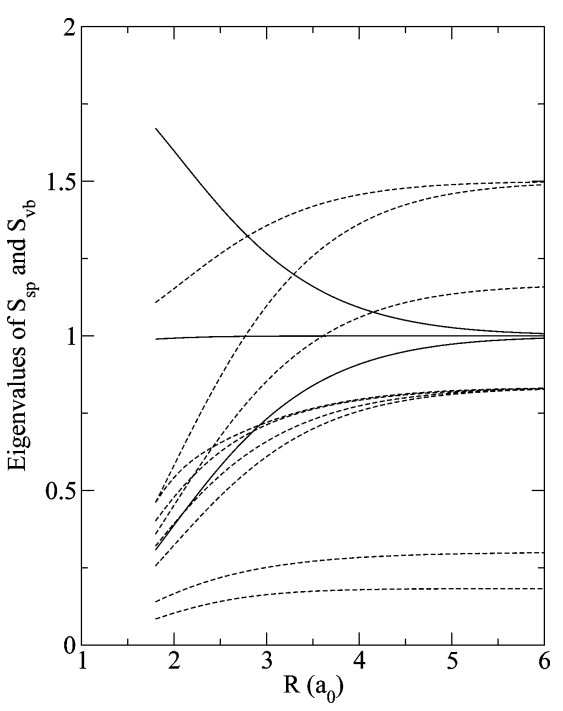

Figure 6. Eigenvalues of the doublet- and quartet-state metric matrices of eq 13 for diatomic $\mathrm{CH}$, constructed employing hydrogen (1s) and carbon $1 s^{2}\left(2 s^{2} 2 p^{2}+2 s 2 p^{3}+2 p^{4}\right)$ multiplet atomic configurations in an optimized $7 \mathrm{~s} 5 \mathrm{p} 3 \mathrm{~d} 2 \mathrm{f} 1 \mathrm{~g}$ valence orbital basis. ${ }^{29}$ The solid lines refer to eigenvalues of the matrix in the orthonormal spectral-product representation, whereas the dashed lines are those obtained in the same configurational basis employing the normalization conventions of the standard tableau functions of valence-bond theory. ${ }^{6,25}$

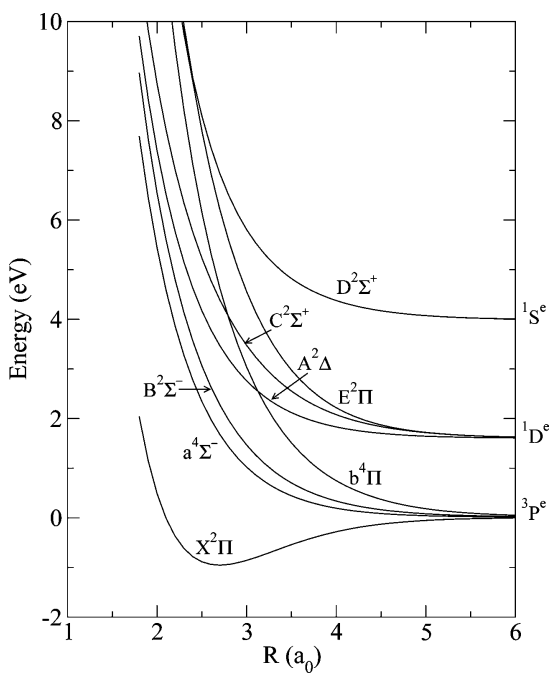

Figure 7. Potential energy curves for diatomic $\mathrm{CH}$ constructed employing minimal valence-shell multiplet carbon $\left(1 \mathrm{~s}^{2} 2 \mathrm{~s}^{2} 2 \mathrm{p}^{2}\right){ }^{3} \mathrm{P}^{\mathrm{e}},{ }^{1} \mathrm{D}^{\mathrm{e}}$, ${ }^{1} S^{e}$ and hydrogen $(1 s)^{2} S^{e}$ atomic eigenstates, providing a representation of $2 \times 15=30$ spectral-product terms. The spectroscopic state labels employed follow conventions from experimental and previously reported theoretical studies. ${ }^{15}$

employed in Figure 6 is too small to exhibit the full redundancy of the basis of eq 4 in this case.

Just as the metric matrices obtained in the valence-bond and spectral-product representations generally differ, so also do the corresponding Hamiltonian matrices. Nevertheless, the energy eigenvalues obtained from the two representations employing identical atomic configurations must be identical, providing a useful test of the algorithms and codes devised to perform the present calculations. In Figure 7 are shown low-lying potential energy curves for $\mathrm{CH}$ obtained in $(1 \mathrm{~s})^{2} \mathrm{~S}^{\mathrm{e}}$ atomic hydrogen and $\left(1 \mathrm{~s}^{2} 2 \mathrm{~s}^{2} 2 \mathrm{p}^{2}\right)^{3} \mathrm{P}^{\mathrm{e}},{ }_{1}^{1} \mathrm{D}^{\mathrm{e}},{ }^{1} \mathrm{~S}^{\mathrm{e}}$ atomic carbon representations. The two sets of potential energy curves obtained from the valence-bond and spectral-product representations of dimension $2 \times 15=$ 30 are found to be identical but to provide generally poor

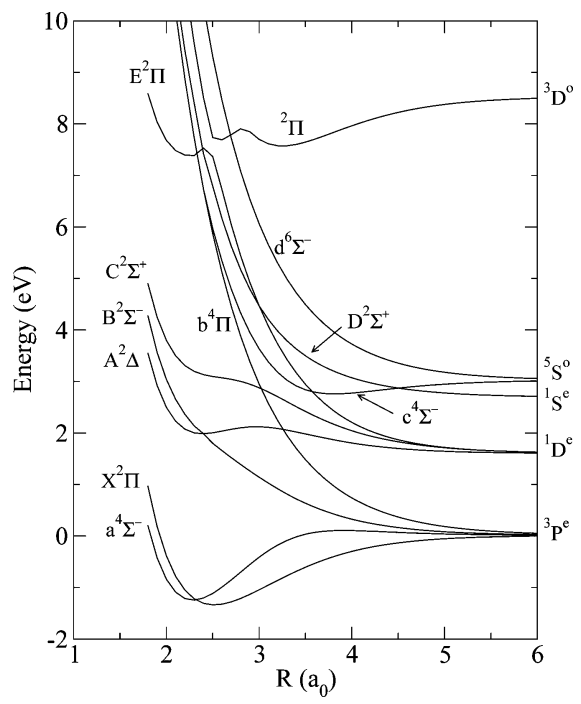

Figure 8. Potential energy curves for diatomic $\mathrm{CH}$ obtained in valenceshell multiplet carbon $1 \mathrm{~s}^{2}\left(2 \mathrm{~s}^{2} 2 \mathrm{p}^{2}+2 \mathrm{~s} 2 \mathrm{p}^{3}+2 \mathrm{p}^{4}\right)$ and hydrogen (1s) atomic eigenstates, providing a representation of $2 \times 55=110$ spectralproduct terms. The origins of the significant differences between these curves and the similarly labeled curves of Figure 7 are discussed in the text. Additional curves dissociating to atomic carbon multiplet states above the ${ }^{3} \mathrm{D}^{\circ}$ limit are calculated but not reported here.

representations of the known accurate values. ${ }^{15}$ Specifically, although the ground state is correctly predicted to be of ${ }^{2} \Pi$ symmetry, the calculated chemical binding energy is much too small, the predicted $\mathrm{A}^{2} \Delta$ state incorrectly falls above the $\mathrm{B}^{2} \Sigma^{-}$ state, and no other chemically bound states are predicted by the representation. Improvements in the quality of the ${ }^{3} \mathrm{P}^{\mathrm{e}},{ }^{1} \mathrm{D}^{\mathrm{e}}$, and ${ }^{1} \mathrm{~S}^{\mathrm{e}}$ carbon-atom multiplet states employed in the spectralproduct representation not reported here are found to have negligible effect on the calculated potential energy curves of Figure 7.

In Figure 8 are shown the results of calculations similar to those of Figure 7, but now including the additional atomic carbon $n=2$ shell multiplet configurations $1 \mathrm{~s}^{2} 2 \mathrm{~s}^{2} \mathrm{p}^{3}$ and $1 \mathrm{~s}^{2} 2 \mathrm{p}^{4}$. The former configuration gives rise to the important ${ }^{5} \mathrm{~S}^{0}$ and ${ }^{3} \mathrm{D}^{\circ}$ atomic carbon states, which have significant effect on the calculated potential energy curves. In particular, the a ${ }^{4} \Sigma^{-}, \mathrm{A}^{2} \Delta$, $\mathrm{B}^{2} \Sigma^{-}$, and $\mathrm{C}^{2} \Sigma^{+}$state curves are significantly lower than those of Figure 7 and in the accepted order, a chemically bound a ${ }^{4} \Sigma^{-}$curve is now obtained, and the ground $X^{2} \Pi$ state is more strongly bound than in Figure 7. The significant lowering of the $a^{4} \Sigma^{-}$state curve is due to the additional ${ }^{4} \Sigma^{-}$configurational state function, which dissociates to the ${ }^{5} \mathrm{~S}^{0}$ atomic carbon state, present in the full valence-shell description of bonding in $\mathrm{CH}$. That is, the well-known $\mathrm{sp}^{3}$ configuration of valence-bond theory appears in the spectral-product representation in the $\left(1 s^{2} 2 s 2 p^{3}\right)^{5} S^{0}$ atomic carbon valence state, which is spectrally concentrated in the $a^{4} \Sigma^{-}$state at the equilibrium interatomic separation. Similarly, the significant lowering of the $\mathrm{A}^{2} \Delta$ and $\mathrm{B}^{2} \Sigma^{-}$state curves correlating with the excited ${ }^{1} \mathrm{D}^{\mathrm{e}}$ atomic carbon state is due to contributions in the spectral-product representation from the high-lying $\left(1 \mathrm{~s}^{2} 2 \mathrm{~s} 2 \mathrm{p}^{3}\right)^{3} \mathrm{D}^{\mathrm{o}}$ atomic carbon state. Additional higher-lying potential energy curves obtained from the calculations arising from the ${ }^{3} \mathrm{P}^{\mathrm{o}},{ }^{1} \mathrm{D}^{\mathrm{o}},{ }^{3} \mathrm{~S}^{\mathrm{o}},{ }^{3} \mathrm{P}^{\mathrm{e}},{ }^{1} \mathrm{D}^{\mathrm{e}}$, and ${ }^{1} \mathrm{~S}^{\mathrm{e}}$ carbon atom multiplets can be analyzed in similar fashion but are not reported here.

Although population-analysis methods can be employed to identify qualitatively the presence of important configurations in calculated molecular wave functions, the atomic spectral concentration of particular atomic components in the $\mathrm{sp}$ 


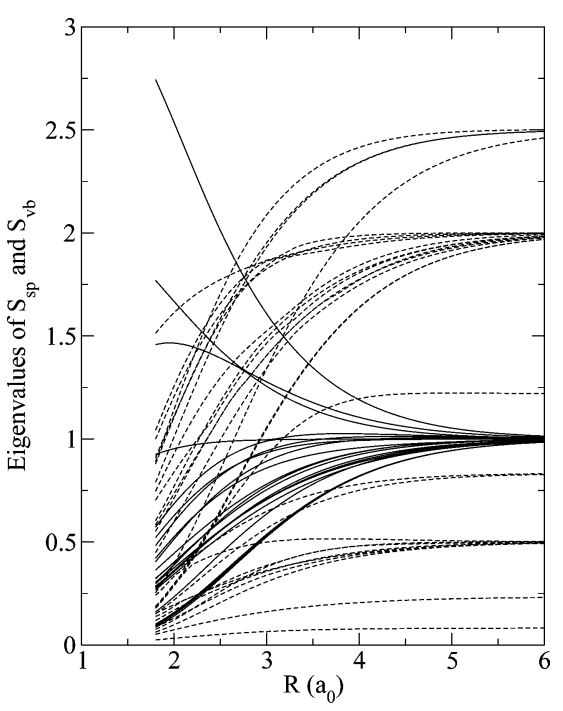

Figure 9. Eigenvalues of the singlet- and triplet-state metric matrices of eq 13 for symmetric collinear $(\mathrm{H}-\mathrm{C}-\mathrm{H})$ methylene arrangements, constructed employing products of valence-shell multiplet carbon $1 s^{2}\left(2 s^{2} p^{2}+2 s 2 p^{3}+2 p^{4}\right)$ and hydrogen (1s) atomic configurations. The solid lines refer to eigenvalues of the matrices constructed in the orthonormal spectral-product representation, whereas the dashed lines are those obtained in the same configurational basis employing the normalization conventions of the standard tableau functions of valencebond theory. 6,25

representation is particularly meaningful in that these states have physical significance and are not subjectively based on particular choices of basis sets, methods of calculation, or analysis techniques. Moreover, the contributions from particular atomicstate products are read off directly and quantitatively from the eigenvectors obtained in the representational basis without further subjective manipulations.

C. Triatomic $\mathbf{C H}_{2}$ Molecule. The methylene molecule has played an important role in the development of accurate quantum chemical computational methodologies ${ }^{16}$ and accordingly is studied here in connection with the metric matrices and atomic spectral compositions of molecular wave functions of interest to the spectral-product development. Calculations similar to those reported above for the $\mathrm{CH}$ molecule help to further clarify aspects of the spectral-product development employing atomic multiplet configurational models for illustrative purposes.

In Figure 9 are shown metric matrix eigenvalues in both valence-bond and spectral-product representations employing the atomic hydrogen $(1 \mathrm{~s})$ and carbon $1 \mathrm{~s}^{2}\left(2 \mathrm{~s}^{2} 2 \mathrm{p}^{2}+2 \mathrm{~s} 2 \mathrm{p}^{3}+2 \mathrm{p}^{4}\right)$ configurations described in the preceding section. In this threeatom, eight-electron case, the spectral-product representation of eq 3 includes $2 \times 2 \times 55=220$ distinct spectral-product terms. The redundancy factor for the corresponding basis of eq 4 is formally $Q=8 ! /(6 ! 1 ! 1 !)=56$, although the closed $1 \mathrm{~s}^{2}$ carbon shell and the absence of higher hydrogen and carbon orbital excitations limits the calculated eigenvalues to a much smaller spectral interval. As in Figure 6 for the $\mathrm{CH}$ molecule, the eigenvalues of Figure 9 are very different in the two representations, with the highly degenerate spectral-product values uniformly approaching unity at large atomic separation and those in the valence-bond representation forming distinct groups associated with the normalizations of standard tableau functions transforming under the different irreducible representations of $S_{n_{t}}{ }^{19}$ Of course, the corresponding Hamiltonian matrices in the two representations also differ, although the energy eigenvalues must be identical when the same orbital configurations are employed in the two developments.

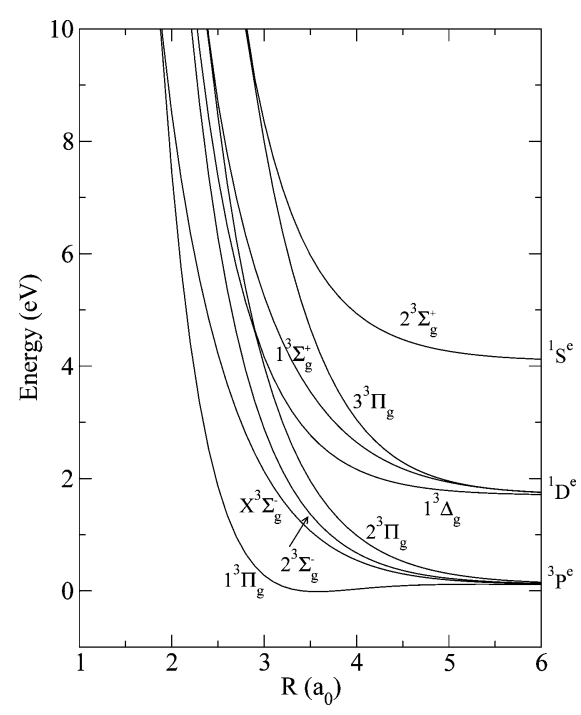

Figure 10. Potential energy curves for triplet methylene states in symmetric collinear $(\mathrm{H}-\mathrm{C}-\mathrm{H})$ arrangements obtained in minimal valence-shell multiplet carbon $\left(1 \mathrm{~s}^{2} 2 \mathrm{~s}^{2} 2 \mathrm{p}^{2}\right)$ and hydrogen (1s) atomic eigenstates, providing a representation of $2 \times 2 \times 15=60$ spectralproduct terms. The ground $\mathrm{X}^{3} \Sigma_{\mathrm{g}}^{-}$state is labeled on basis of experimental and previously reported theoretical studies, ${ }^{16}$ whereas the other states are given sequential numerical labels.

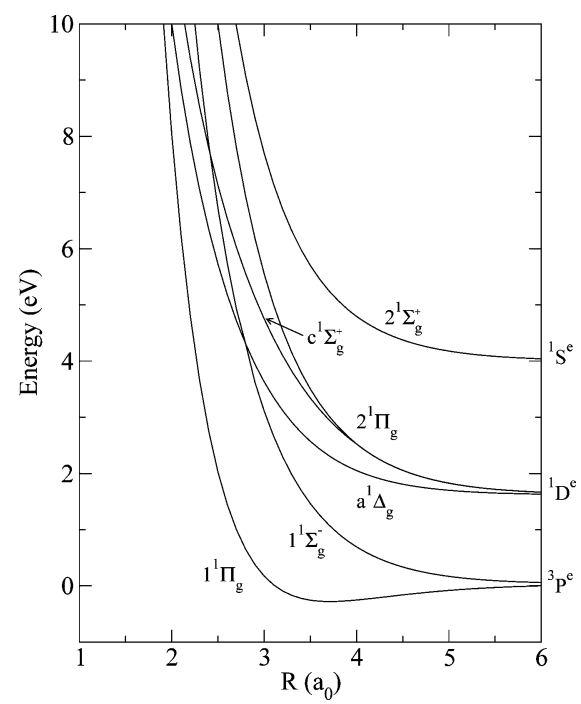

Figure 11. Potential energy curves for singlet methylene states in symmetric collinear $(\mathrm{H}-\mathrm{C}-\mathrm{H})$ arrangement obtained in minimal valence-shell multiplet carbon $\left(1 \mathrm{~s}^{2} 2 \mathrm{~s}^{2} 2 \mathrm{p}^{2}\right)$ and hydrogen (1s) atomic configurations, providing a representation of $2 \times 2 \times 15=60$ spectralproduct terms. The two states given spectroscopic-state labels follow conventions from experimental and previously reported theoretical studies, ${ }^{16}$ whereas the other states are given sequential numerical labels.

In Figures 10 and 11 are shown triplet- and singlet-state potential energy curves for methylene in symmetric collinear $(\mathrm{H}-\mathrm{C}-\mathrm{H})$ arrangements obtained in the multiplet carbon $\left(1 s^{2} 2 s^{2} 2 p^{2}\right)$ and hydrogen (1s) atomic configurations, proving a reduced spectral-product representation of $2 \times 2 \times 15=60$ atomic-product states in this case. The lowest-lying potential curves in this minimal valence-multiplet representation are seen to be very weakly bound ${ }^{3} \Pi_{\mathrm{g}}$ and ${ }^{1} \Pi_{\mathrm{g}}$ states, whereas the correct lowest-energy triplet and singlet states in symmetric collinear $\mathrm{CH}_{2}$ arrangement are known to be the $\mathrm{X}^{3} \Sigma_{\mathrm{g}}^{-}$and $\mathrm{a}^{1} \Delta_{\mathrm{g}}$ states. ${ }^{16}$ Additionally, all other curves in Figures 10 and 11 are seen to be strongly nonbonding. Clearly, the minimal multiplet representation fails to provide even qualitatively correct descriptions 


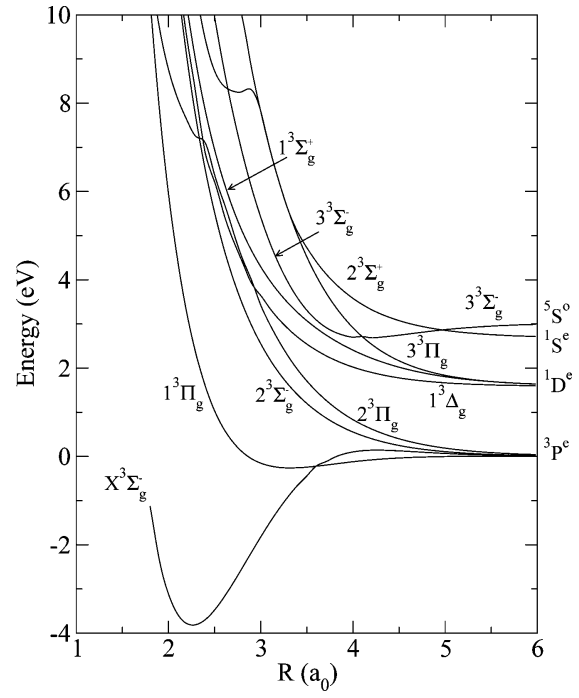

Figure 12. Potential energy curves for triplet methylene states in symmetric collinear $(\mathrm{H}-\mathrm{C}-\mathrm{H})$ arrangements obtained in valence-shell multiplet carbon $1 \mathrm{~s}^{2}\left(2 \mathrm{~s}^{2} 2 \mathrm{p}^{2}+2 \mathrm{~s} 2 \mathrm{p}^{3}+2 \mathrm{p}^{4}\right)$ and hydrogen (1s) atomic configurations, providing a representation of $2 \times 2 \times 55=220$ spectralproduct terms. The origins of the significant differences between these curves and the similarly labeled curves of Figure 10 are discussed in the text. Additional higher-lying and higher-multiplicity states are calculated but not reported here.

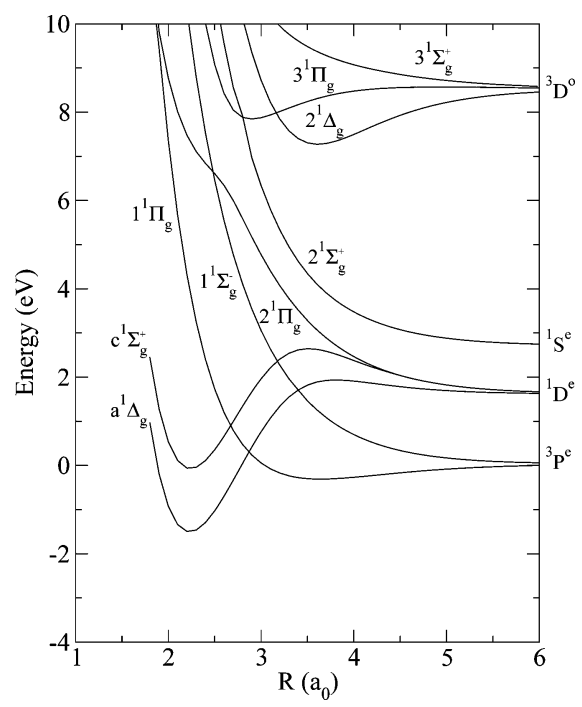

Figure 13. Potential energy curves for singlet methylene states in symmetric collinear $(\mathrm{H}-\mathrm{C}-\mathrm{H})$ arrangement obtained in valence-shell multiplet carbon $1 \mathrm{~s}^{2}\left(2 \mathrm{~s}^{2} 2 \mathrm{p}^{2}+2 \mathrm{~s} 2 \mathrm{p}^{3}+2 \mathrm{p}^{4}\right)$ and hydrogen (1s) atomic states, providing a representation of $2 \times 2 \times 55=220$ spectral-product terms. The origins of the significant differences between these curves and the similarly labeled curves of Figure 11 are discussed in the text. Additional higher-lying and higher-multiplicity states are calculated but not reported here.

of the potential energy curves in $\mathrm{CH}_{2}$. As in the case of $\mathrm{CH}$ indicated above, improvements in the quality of the ${ }^{3} \mathrm{P}^{\mathrm{e}},{ }^{1} \mathrm{D}^{\mathrm{e}}$, and ${ }^{1} \mathrm{~S}^{\mathrm{e}}$ carbon atom multiplets employed in calculations not reported here are found to have little effect on the results of Figures 10 and 11.

In Figures 12 and 13 are shown potential energy curves for the states of Figures 10 and 11, now constructed by employing the 55 carbon atom $1 \mathrm{~s}^{2}\left(2 \mathrm{~s}^{2} 2 \mathrm{p}^{2}+2 \mathrm{~s} 2 \mathrm{p}^{3}+2 \mathrm{p}^{4}\right)$ valence-shell configurations, providing $2 \times 2 \times 55=220$ individual atomicproduct terms in the spectral-product representation of eq 3 in this case. As in the case of $\mathrm{CH}$, there are significant changes in the $\mathrm{CH}_{2}$ potential curves of Figures 12 and 13 relative to those of Figures 10 and 11. Specifically, the $\mathrm{X}^{3} \Sigma_{\mathrm{g}}^{-}$state in Figure 12 is seen to become the lowest energy state, the $\mathrm{a}^{1} \Delta_{\mathrm{g}}$ state in Figure 13 becomes the lowest-lying single state, and the $\mathrm{c}^{1} \Sigma_{\mathrm{g}}^{+}$ state there becomes the first excited singlet, in accord with the results of accurate calculations. ${ }^{16}$ The lowering of the $\mathrm{X}^{3} \Sigma_{\mathrm{g}}^{-}$ potential curve is a consequence of the additional ${ }^{3} \Sigma_{\mathrm{g}}^{-}$configuration arising from the presence of the ${ }^{5} \mathrm{~S}^{\circ}$ carbon atom state in the larger representation, whereas the lowering of the $\mathrm{a}^{1} \Delta_{\mathrm{g}}$ and $\mathrm{c}^{1} \Sigma_{\mathrm{g}}^{+}$states is due to the indicated additional states dissociating to the high-lying ${ }^{3} \mathrm{D}^{\circ}$ carbon atom state in the larger atomic representation employed. As in the case of $\mathrm{CH}$ indicated above, the $\mathrm{sp}^{3}$ configuration in atomic carbon through its appearance in the ${ }^{5} \mathrm{~S}^{\circ}$ and ${ }^{3} \mathrm{D}^{\circ}$ atomic states plays a significant role in the nature of chemical binding in methylene, findings based on direct inspection of the molecular eigenvectors in the spectral-product representation.

\section{Discussion and Concluding Remarks}

The atomic spectral-product approach to ab initio molecular electronic structures reported here attempts to provide a fresh perspective on such calculations based on products of formally complete sets of atomic eigenfunctions. The theoretical development presented in section II demonstrates the equivalence between enforcement of electron antisymmetry either prior to or subsequent to Hamiltonian matrix evaluation in such an atomic spectral-product representation. In the more familiar case of prior enforcement of electron antisymmetry in the manyelectron representation, Hamiltonian matrix elements depend on the positions of all the atoms in the aggregate, and require repeated evaluations in construction of potential energy surfaces. A linearly independent subspace of this redundant representation must generally be isolated to avoid encountering computational instabilities in obtaining molecular energy eigenfunctions and eigenvalues. In contrast, the post-antisymmetrization approach employs a complete but not overcomplete atomic-eigenstateproduct representation in the absence of term-by-term electron antisymmetrization, providing Hamiltonian matrix elements that can be evaluated once and for all and retained for repeated polyatomic applications. There remains the task in this approach of isolating the totally antisymmetric subspace of the spectralproduct representation to obtain the physically significant molecular eigenstates.

The matrix representing the total antisymmetrizer in the spectral-product basis provides a method for isolating the totally antisymmetric subspace of the representation by unitary transformation of the Hamiltonian matrix after its evaluation. This unitary transformation approach to antisymmetrization provides an alternative to the more commonly employed prior global antisymmetrization. It is seen from the development of section II that the antisymmetrizer matrix is, in fact, equivalent to the metric matrix of the antisymmetrized form of the spectralproduct representation. Accordingly, there is a close connection between isolating the totally antisymmetric representation of a many-electron atomic-product basis with the canonical orthogonalization of its explicitly antisymmetric form.

The computational applications to simple molecules $\left(\mathrm{H}_{2}, \mathrm{H}_{3}\right.$, $\mathrm{CH}, \mathrm{CH}_{2}$ ) reported in section III illustrate the role of the eigenvalue spectrum of the metric matrix in estimating closure in the spectral-product representation, and in correspondingly identifying redundancy in its explicitly antisymmetrized form. The allowable range of the eigenvalue spectrum of the antisymmetrizer employed in the spectral-product basis is seen to be determined by the number of electrons in each atom and by their total sum. Convergence of the eigenvalues to the extreme 
points of the allowable range in the case of triatomic hydrogen $\mathrm{H}_{3}$ is seen to correspond to the separation of totally antisymmetric and non totally antisymmetric states in the spectralproduct basis, or, equivalently, to the separation of linearly independent and linearly dependent states in its antisymmetrized form. The introduction of additional terms in atomic-product representations, typified by charge-transfer terms in the case of diatomic hydrogen $\mathrm{H}_{2}$, is seen to disrupt this simple picture, and to extend the allowable range of the eigenvalue spectrum of the antisymmetrizer to accommodate the additional basisset redundancy introduced. The metric matrix appears to provide a device for developing suitable atomic spectral-product representations entirely in the absence of the molecular electronic energy calculations commonly employed in devising orbital basis sets for use in more conventional calculations.

The illustrative molecular energy calculations of the simple molecules studied $\left(\mathrm{H}_{2}, \mathrm{CH}, \mathrm{CH}_{2}\right)$ reported in section III demonstrate the capabilities of the Slater-orbital- and valencebond-based algorithms and codes devised for this purpose, as well as the nature of a descriptor of molecular electronic structures the development provides. Calculations of the ground and excited-state potential energy curves in $\mathrm{H}_{2}$ dissociating to the $n=1$ and $n=2$ atomic limits, obtained from straightforward diagonalizations of singlet and triplet configuration-interaction energy matrices constructed with Slater-orbital basis sets, are found to be in good accord with the most accurate calculations available, suggesting the codes devised should be applicable to other molecules more generally. Employing simple multiplet models as examples, the important role of the $2 \mathrm{~s} 2 \mathrm{p}^{3}$ configuration in atomic carbon is understood from a new perspective provided by the spectral-product development. Specifically, the contributions to molecular $\mathrm{CH}$ and $\mathrm{CH}_{2}$ eigenstates from ${ }^{5} \mathrm{~S}^{\circ}$ and ${ }^{3} \mathrm{D}^{\circ}$ atomic carbon states which arise specifically from the $2 \mathrm{~s} 2 \mathrm{p}^{3}$ configuration are seen to have significant qualitative and quantitative effects on the positions and shapes of selected potential energy curves. The quantitative perspective provided by the atomic-product compositions of molecular electronic wave functions in the spectral-product representation involves the physically significant many-electron atomic eigenstates of the bonding atoms. Accordingly, it would seem the spectralproduct representation provides both a potentially useful computational and conceptual and basis for studies of molecular electronic eigenstates.

Additional calculations not reported here involving $\mathrm{H}, \mathrm{B}, \mathrm{C}$, $\mathrm{N}, \mathrm{O}$, and $\mathrm{F}$ atom-containing compounds largely support and elaborate the foregoing general conclusions. Studies in progress are now focused on development of significantly more efficient and robust computational methods for performing spectralproduct calculations, use of larger Slater-orbital representations in such computational applications, efficient means of isolating the required totally antisymmetric subspaces, and related developmental matters.
Acknowledgment. The financial support of the Air Force Research Laboratory is gratefully acknowledged. We thank Drs. J. A. Sheehy and S. L. Elbert for assistance and advice during the early stages of the investigation, and Professor J. A. McCammon for his kind hospitality and support throughout.

\section{References and Notes}

(1) Eisenschitz, H.; London, F. Z. Phys. 1930, 60, 491-527.

(2) Hund, F. Z. Phys. 1927, 40, 742-764.

(3) Mulliken, R. Phys. Rev. 1928, 32, 186-222.

(4) Pauling, L. Proc. Natl. Acad. Sci. U.S.A. 1928, 14, 359-362.

(5) Slater, J. C. Phys. Rev. 1931, 38, 1109-1144.

(6) Hehre, W. J.; Radom, L.; Schleyer, P. V. R.; Pople, J. A. Ab Initio Molecular Orbital Theory; John Wiley and Sons: New York, 1986.

(7) Shaik, S. S.; Hiberty, P. C. A Chemist's Guide to Valence-bond Theory; Wiley-Interscience: New York, 2008.

(8) Moffitt, W. Proc. Roy. Soc., London A 1951, 210, 245-268.

(9) Langhoff, P. W. J. Phys. Chem. 1996, 100, 2974-2984.

(10) Langhoff, P. W.; Hinde, R. J.; Boatz, J. A.; Sheehy, J. A. Chem. Phys. Lett. 2002, 358, 231-236.

(11) Langhoff, P. W.; Boatz, J. A.; Hinde, R. J.; Sheehy, J. A. In LowLying Potential Energy Surface; Hoffmann, M. R., Dyall, K. G., Eds.; ACS Symposium Series 828; American Chemical Society: Washington, DC, 2002; Chapter 10, pp 221-237.

(12) Langhoff, P. W.; Boatz, J. A.; Hinde, R. J.; Sheehy, J. A. In Fundamental World of Quantum Chemistry: A Tribute to the Memory of Per-Olov Löwdin; Brändas, E., Kryachko, E. S., Eds.; Kluwer Academic: Dordrecht, The Netherlands, 2004; Volume 3, pp 97-114.

(13) Langhoff, P. W.; Boatz, J. A.; Hinde, R. J.; Sheehy, J. A. J. Chem. Phys. 2004, 121, 9323-9342.

(14) Langhoff, P. W.; Boatz, J. A.; Hinde, R. J.; Sheehy, J. A. Theor. Chem. Acc. 2008, 120, 199-213.

(15) Kalemos, A.; Mavridis, A.; Metropoulos, A. J. Chem. Phys. 1999, $111,9536-9548$

(16) Kalemos, K; Dunning, T. H., Jr.; Mavridis, A.; Harrison, J. F. Can. J. Chem. 2004, 82, 684-693.

(17) McWeeny, R. Methods of Molecular Quantum Mechanics, 2nd ed.; Academic: London, U.K, 1989.

(18) Bethe, H. A.; Salpeter, E. E. Quantum Mechanics of One- and TwoElectron Atoms; Springer-Verlag: Berlin, DE, 1957.

(19) Hamermesh, M. Group Theory; Addison-Wesley: Reading, MA, 1962.

(20) Condon, E. U.; Shortley, G. H. The Theory of Atomic Spectra; University Press: Cambridge, U.K., 1963.

(21) Courant, R.; Hilbert, D. Methods of Mathematical Physics; Interscience: New York, 1966; Vol. I, Chapter II.

(22) Chisholm, C. D. H. Group Theoretical Techniques in Quantum Chemistry; Academic Press: London, U.K, 1976; Chapter 6.

(23) Akhiezer, N. I.; Glazman, I. M. Theory of Linear Operators in Hilbert Space; Ungar: New York, 1961; Vol. I.

(24) Szabo, A.; Ostlund, N. S. Modern Quantum Chemistry: Introduction to Advanced Electronic Structure Theory; MacMillan: London, U.K., 1982; pp 142-145.

(25) Gallup, G. A. Valence Bond Methods: Theory and Applications; Cambridge University Press: New York, 2002.

(26) Fernádez Rico, J.; Lopez, R.; Ema, I.; Ramírez, G. J. Comput. Chem. 2004, 25, 1987-1994.

(27) Porter, R. N.; Karplus, M. J. Chem. Phys. 1964, 40, 1105-1115.

(28) Shull, H.; Löwdin, P.-O. J. Chem. Phys. 1955, 23, 1362.

(29) Ema, I.; Garcia de la Vega, J. M.; Ramírez, G; López,; Fernádez Rico, R. J.; Meissner, H.; Paldus, J. J. Comput. Chem. 2003, 24, 859-868.

(30) Fantz, U.; Wunderlich, D. Franck-Condon Factors, Transition Probabilities and Radiative Lifetimes for Hydrogen Molecules and Their Isotopomeres; Report INDC(NDS)-457; International Atomic Energy Agency: Vienna, Austria, 2004.

JP901427X 